\title{
Polymeric Nanomaterials as Electrolyte and Electrodes in Supercapacitors
}

\author{
João P. C. Trigueiro, Raquel S. Borges, Rodrigo L. Lavall, Hallen D. R. Calado, and Glaura G. Silva（ $₫)$ \\ Departamento de Química, Instituto de Ciências Exatas, Universidade Federal de Minas Gerais, CEP 31270-901, Belo Horizonte, Brazil \\ Received: 13 April 2009 / Revised: 17 August 2009 / Accepted: 17 August 2009 \\ CTsinghua University Press and Springer-Verlag 2009. This article is published with open access at Springerlink.com
}

\begin{abstract}
The energy challenge requires a broad range of options for energy harvesting, storage, and conversion. We have produced polymeric coatings by spraying, to be used as electrolyte and electrodes in a flexible electrochemical double layer capacitor. A thermoplastic polyurethane and a low molecular weight block copolyether were employed with $\mathrm{LiClO}_{4}$ to prepare solid polymeric electrolytes. Carbon black (CB) and multi-walled carbon nanotubes (MWNTs) were dispersed in the polymer blend electrolyte to produce nanostructured composite electrodes. The conductivities increased with the addition of block copolyether and carbon nanotubes to the electrolyte and electrode, respectively. Scanning electron microscopy (SEM) and atomic force microscope (AFM) images of the nanocomposite electrodes showed nanoagglomerates of CB connected by carbon nanotubes. The solid supercapacitor prepared with these new materials as electrolyte and electrodes showed superior performance to other similar systems. The resulting safe and flexible multilayer device can meet the requirements of modern devices.
\end{abstract}

\section{KEYWORDS}

Polymeric electrolyte blend, nanocomposite electrode, carbon black, carbon nanotube, supercapacitor

Electrochemical capacitors or supercapacitors [1, $2]$, using the electric double layer charge at the electrode/electrolyte interface of a highly porous electrode, can have an important role in new technologies. The properties of supercapacitors complement the deficiencies of other power sources, such as batteries and fuel cells [3].

Nanostructured carbon-based materials have been used in electrodes for electrochemical double layer capacitors (EDLC). The relationship between the surface area, total pore volume, average pore size, and the pore size distribution of the materials has a strong influence on the electrochemical characteristics of the resulting capacitor $[3,4]$. In such devices, activated carbons, carbon black or carbon nanotube composites have been employed as electrodes [3 -6], mainly with liquid electrolytes [7]. Usually, the electrolytes employed in the electrochemical capacitors are acids, bases or salts dissolved in aqueous or organic solvents. Ionic liquids have also been investigated in supercapacitors [8-10]. The use of corrosive liquid electrolytes may cause dangerous leakages, which decrease the safety and lifetime of the capacitors $[11,12]$. To reduce problems associated

Address correspondence to glaura@qui.ufmg.br 
with the management of corrosive ionic conductors, as well as to allow the preparation of thin film cells with high reliability, the use of solid polymer electrolytes (SPE) has been proposed [11, 13-16].

The performance of carbon-based polymer supercapacitors is closely associated with the characteristics of the materials used, such as new carbonaceous and electrolytes. The characteristics of the polymer electrolyte need to be tailored with the goal of increasing conductivity under specific experimental conditions. A degree of matching between the carbonaceous pore size and the microstructural arrangement of the ions in the electrolyte seems to be more important in terms of capacitor performance than the pore size distribution itself [9]. The polymer chains of an SPE did not show long range displacement during ionic migration, leading to the concept of "immobile solvent" [17]. Although there is a true coordination of cations by oxygen atoms, the anions are more loosely held in the bulk material. Micropores $(0-1 \mathrm{~nm})$ or small mesopores $(\sim 2 \mathrm{~nm})$ may not be accessible to the ions in this case. Therefore, the strategy is to disperse the carbonaceous particles throughout the entire polymer electrolyte film, keeping low filler content to take advantage of the surface area, which is directly exposed to the polymer in solid interfaces. Moreover, a mixture of a low molecular weight polymer with the main polymer matrix allows the preparation of polymer blends, which improve wetability of the carbonaceous material, and also enhancement of ionic access to the range of mesopores $(\sim 2-50 \mathrm{~nm})$ constituting the double layer.

Carbon black (CB) is a particulate form of carbon, that can be obtained-depending on the preparation procedure - with a diameter range from 10 to 100 $\mathrm{nm}$, forming extremely porous materials with large surfaces areas (100-2000 m²/g) [3, 18]. Carbon blacks with small diameter and large surface area are suitable as fillers to improve electrical conductivity and to create suitable microstructure for charge storage.

Carbon nanotubes (CNTs) are opening up exciting new opportunities in several fields [19], including storage devices, such as supercapacitors [4, 5, 8, 9, 20-23]. The production of highly conductive pathways, at very low concentration of nanofillers, is one of the most useful properties of CNTs in supercapacitors. The preparation of mats or shape-engineerable forms may allow the smart exploitation of their surface area [22].

Our group has recently reported a study of materials for electrochemical capacitors, using $\mathrm{CB}$ and poly(ethylene oxide) PEO mixed with a polyether copolymer of low molar mass [16], prepared by spray coating. In this paper we describe two improvements to the previous procedure: the attempt to take advantage of a new kind of carbonaceous material, namely CNT, to increase electronic conductivity and the use of a commercial thermoplastic polyurethane (TPU) giving higher dimensional stability at higher temperatures without loss of ionic conductivity.

\section{Experimental}

The materials used to prepare SPE and composite electrodes (CE) by spray casting were TPU (Irogran PS455-203 from Huntsman), $\mathrm{LiClO}_{4}$ (Aldrich), copolymer poly(propylene glycol)-blockpoly(ethylene glycol)-block-poly(propylene glycol) (COP) (Aldrich, $M_{\mathrm{n}}=2000 \mathrm{~g} / \mathrm{mol}-$ supplier data), CB (Cabot, Black Pearls 2000, $1500 \mathrm{~m}^{2} / \mathrm{g}$, particle size of $12 \mathrm{~nm}$-supplier data), multi-walled carbon nanotubes (MWNT) (CNT Co. Ltd., Incheon, Korea), and tetrahydrofuran (THF) (Synth). Carbon black was washed in water and THF in an ultrasonic bath for $3 \mathrm{~h}$ and then oven dried. All other reagents were used as received.

The specific superficial area for $C B$ was determined previously to be $1680 \mathrm{~m}^{2} / \mathrm{g}$ [16]. The BET isotherm indicated the presence of micropores $(<2$ $\mathrm{nm})$ and mesopores (2-50 nm). MWNTs synthesized by chemical vapor deposition were employed in this work. The range of nanotube diameter and length given by the supplier was 10-40 nm and 5$20 \mu \mathrm{m}$, respectively. The MWNTs were characterized by thermogravimetry (TG) and scanning electron microscopy (SEM). TG was carried out with an SDT 2960 TA Instruments in air atmosphere with a heating rate of $5{ }^{\circ} \mathrm{C} / \mathrm{min}$. SEM images were obtained with a Jeol JSM 840A for the MWNTs and the composites, without any coating of the samples.

The electrolyte blends BE23 and BE38, 
respectively, were prepared from TPU with 23 $w t \%$ or $38 w t \%$ of COP, and $20 w t \% \mathrm{LiClO}_{4}$. The polymers were dissolved in THF under magnetic agitation. After dissolution of the polymers, the salt was added and mixed by agitation, to produce the final electrolyte blends. The composite electrodes (CE) were prepared using the blend of TPU and 38 $w t \%$ of COP, as polymeric matrix, mixed with $4 \mathrm{wt} \%$ of MWNT and $8 \mathrm{wt} \%$ of carbon black as fillers; the resulting $\mathrm{CE}$ is denoted $\mathrm{CE} 4 / 8$. The suspensions for preparing electrode samples were sonicated for one hour to produce a high dispersion of $\mathrm{CB}$ and MWNT in the polymer blend. The solutions or suspensions were sprayed on stainless steel substrates. The solvent evaporation was carried out under vacuum, for a minimum of $72 \mathrm{~h}$. The thickness of the layers was determined using a Mitutoyo digital micrometer, with a precision of $1 \mu \mathrm{m}$.

Differential scanning calorimetry (DSC) was carried out with a TA Instruments 2920 DSC with a heating/cooling/heating protocol between $-100^{\circ} \mathrm{C}$ and $200{ }^{\circ} \mathrm{C}$ at heating rate of $10{ }^{\circ} \mathrm{C} / \mathrm{min}$, under He atmosphere $(50 \mathrm{~mL} / \mathrm{min})$. A second DSC heat scanning was used to determine phase transitions.

Electrical measurements were performed with an Eco Chemie potentiostat/impedance frequency analyzer Autolab PGSTAT 30. An experimental cell including two stainless steel disk electrodes (collectors) was used for the measurements of conductivity of electrolytes and composite electrodes in the temperature range $25^{\circ} \mathrm{C}$ to $95{ }^{\circ} \mathrm{C}$. The measurements were accomplished over the frequency range $1 \mathrm{~Hz}$ to $5 \times 10^{5} \mathrm{~Hz}$ with $50 \mathrm{mV}$ amplitude.

For the capacitor analysis, complete cells were made by preparing two elements, consisting of a $\mathrm{C}: \mathrm{CE}$ configuration ( $\mathrm{C}=$ collector), which were set up to sandwich an SPE film (BE38) under low pressure. The electrochemical and $\mathrm{AC}$ electrical properties were characterized at $25{ }^{\circ} \mathrm{C}$ and $45^{\circ} \mathrm{C}$. The frequency range employed was from $1 \times 10^{-3}$ $\mathrm{Hz}$ to $1 \times 10^{6} \mathrm{~Hz}$ at $0 \mathrm{~V}$, with $5 \mathrm{mV}$ amplitude, for the impedance measurements. The electrical parameters were deduced using an equivalent circuit program, EQUIVCRT (B. A. Boukamp) or the free Zview version $2.8 \mathrm{~d}$ (demonstration mode) from Scribner Associates, Inc. To check the capacitance data, cyclic voltammetry $(\mathrm{CV})$ for the EDLC was carried out in the voltage range $-1.0 \mathrm{~V}$ to $+1.4 \mathrm{~V}$ at $2 \mathrm{mV} / \mathrm{s}$, at $25^{\circ} \mathrm{C}$ and $45^{\circ} \mathrm{C}$.

\section{Results and discussion}

SEM analysis of MWNT shows (Fig. 1) an open structure consisting of entangled bundles, with diameters between 50 and $200 \mathrm{~nm}$. The BET specific surface area, measured by nitrogen adsorption, is 136 $\mathrm{m}^{2} / \mathrm{g}$ [24]. The MWNT material presents a fraction of residual metal of approximately $6 \mathrm{wt} \%$ (calculated as $\sim 70 \%$ of the final residue weight, corresponding to either $\mathrm{Ni}, \mathrm{Co}$, or Fe metallic oxides), as shown by the thermogravimetric residue of $\sim 9 \mathrm{wt} \%$ in Fig. 1. The decomposition occurs in two stages, with $584{ }^{\circ} \mathrm{C}$ being the temperature of maximum decomposition rate determined in the TG derivative curve (DTG). This two-stage decomposition is associated with the catalytic effect of the metal particles on the nanotube degradation, and the presence of defective material which decomposes first [25]. The TG and SEM results indicated that the CNT used in this work can be considered moderately pure and of good structural quality [26].

The main thermal properties from the DSC second heating scan curves for the polymeric matrices TPU and COP, electrolytes BE23 and BE38 and CE4/ 8 composite electrode are summarized in Table 1 . The TPU presents the typical segmented structure, with $T_{\mathrm{g}}{ }^{1}$ associated with a soft segments amorphous phase, and $T_{\mathrm{g}}{ }^{2}$ and $T_{\mathrm{m}}$ as the thermal

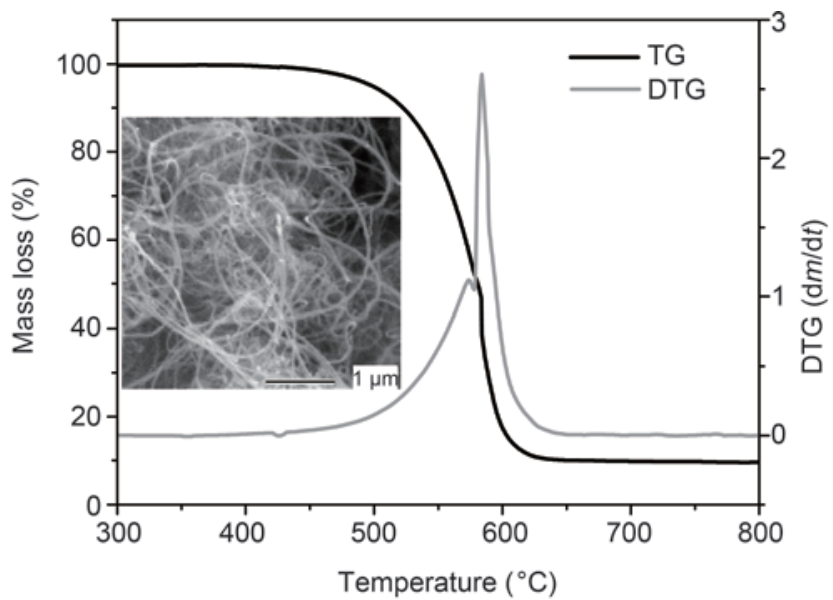

Figure 1 Thermogravimetric (TG) and derivative of TG (DTG) curves for MWCNT at $5{ }^{\circ} \mathrm{C} / \mathrm{min}$ in air. Inset: SEM image 
Table 1 Main thermal data (from DSC second heating curves) for the two polymeric matrices TPU and COP, the electrolytes BE23 and BE38 and the composite CE4/8

\begin{tabular}{c|c|c|c|c}
\hline Sample & $\begin{array}{c}T_{\mathrm{g}}{ }^{2} \\
\text { (mid point) }\left({ }^{\circ} \mathrm{C}\right)\end{array}$ & $\begin{array}{c}T_{\mathrm{g}}^{\mathrm{t}} \\
\text { (mid point) }\left({ }^{\circ} \mathrm{C}\right)\end{array}$ & $\begin{array}{c}T_{\mathrm{m}} \\
\text { (peak) }\left({ }^{\circ} \mathrm{C}\right)\end{array}$ & $\begin{array}{c}\Delta H_{\mathrm{m}} \\
(\mathrm{J} / \mathrm{g})\end{array}$ \\
\hline $\mathrm{TPU}$ & -35 & 40 & 131 (broad) & 4.2 \\
\hline $\mathrm{COP}$ & -71 & - & 10 & 53 \\
\hline $\mathrm{BE} 23$ & -33 to $-20^{*}$ & $\sim 50$ & - & - \\
\hline BE38 & -27 to $-11^{*}$ & $\sim 50$ & - & - \\
\hline $\mathrm{CE} 4 / 8$ & -4 to $2 *$ & - & - & - \\
\hline
\end{tabular}

Variable results from triplicate

events of the rigid segments arrangement [27]. The semicrystalline copolymer showed a low $T_{g^{\prime}}$ probably related to the polyethylene glycol PPG block, and a low $T_{\mathrm{m}}$ related to the polyethylene glycol block [28]. Variable results were obtained from the replicate analysis of electrolytes and composites. This may be associated with heterogeneity of the samples. However, it is possible to extract some important information from Table 1: (1) blend electrolytes show two $T_{\mathrm{g}}$ values, but did not exhibit a melting event, or the very low temperature glass transition of the individual copolymer. This is a good indication of blend miscibility and salt interaction with both TPU and COP; (2) The single $T_{\mathrm{g}}$ value found for the composite confirms the miscibility of TPU and COP components; (3) For both electrolytes and composite, the addition of salt or salt + fillers produces increase in $T_{\mathrm{g}}$. For the electrolyte systems, where lithium cations coordinate to oxygen atoms, the formation of a more rigid amorphous structure is expected [16]. The further increase in $T_{\mathrm{g}}$ with filler addition is a consequence of the jammed microstructure produced by the carbonaceous network [29].

Measurements of the conductivity as a function of temperature were performed after heating to $95^{\circ} \mathrm{C}$ and subsequent cooling. The Arrhenius plots presented in Fig. 2 show that the differences in conductivity between BE23 and BE38 are of one order of magnitude over the complete temperature range. Moreover, the composite with carbonaceous fillers (CE4/8) shows a further increase in conductivity of half an order of magnitude, and exhibits approximately constant conductivity with respect to temperature. This composite is significantly more conductive than the

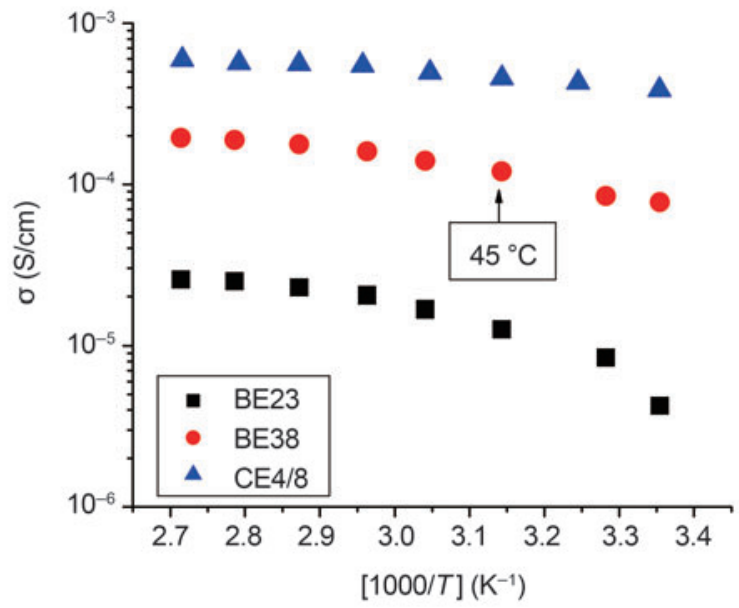

Figure 2 Arrhenius plot for polymer electrolytes prepared with a blend of TPU, 23 wt\% (BE23) and $38 w t \%$ (BE38) of copolymer and $20 \mathrm{wt} \% \mathrm{LiClO}_{4}$; composite from the BE38 and $4 \mathrm{wt} \% \mathrm{MWCNT}+8$ wt\% carbon black (CE4/8)

one used in our previous work [16].

For randomly dispersed cylinders with aspect ratio equal to 10, the theoretical percolation threshold is $12 \mathrm{vol} \%$ [30]. Therefore, this was the concentration of filler added to produce the electrode composite. The MWNT can be considered to enhance the electronic conductivity whilst the $\mathrm{CB}$ aggregates provide higher surface area for charge storage. Figure 3 shows the SEM and AFM images of the composite. It can be observed that $\mathrm{CB}$ aggregates are, to some extent, connected by bundles of MWNT. Different ratios of MWNT were tested. The $4 \mathrm{wt} \%$ concentration of MWNT is the optimized value, under our experimental conditions, because smaller contents lead to less conducting materials and higher contents show nanotube aggregation, and did not improve composite conductivity.

A double layer capacitor with CE4/ 8 electrode films sandwiching a micrometric coating of BE38 was set up between steel current collectors. Typically, the device was designed with thickness of $\sim 150 \mu \mathrm{m}$ and area of $0.685 \mathrm{~cm}^{2}$. The complete impedance response of the capacitor was tested at $25{ }^{\circ} \mathrm{C}$ and $45{ }^{\circ} \mathrm{C}$, as presented in Fig. 4(a). The partial semi-circle at high frequency in the inset to Fig. 4(a), may be associated with the resistive and capacitive behavior within the composite electrode. The overall semi-circle was not observed for this coating, because of the apparatus limitations. The second semi-circle at intermediate 

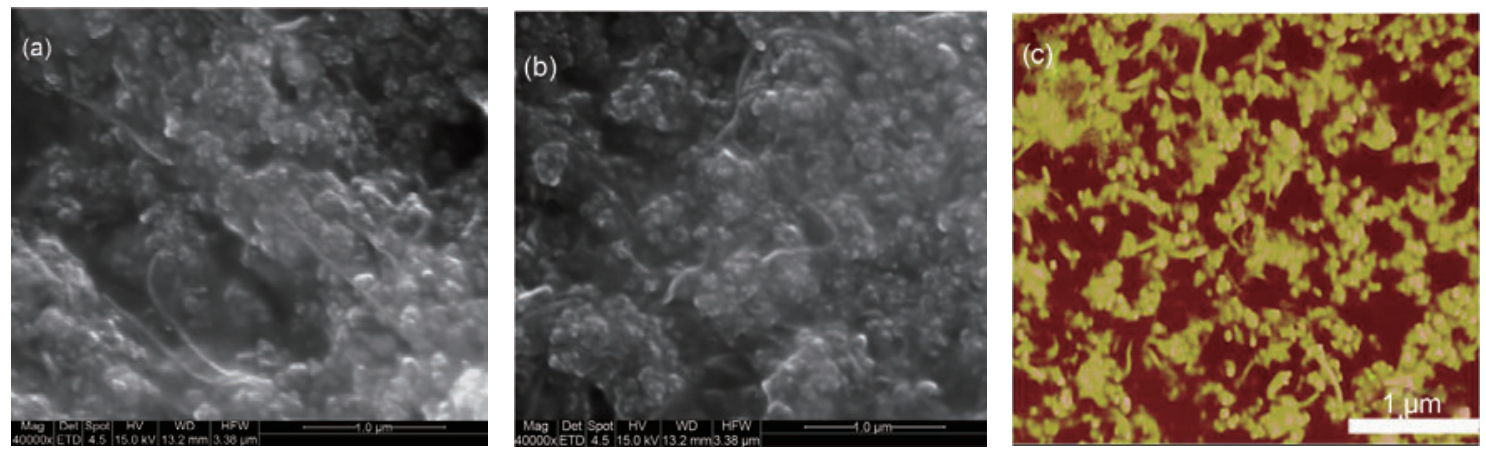

Figure 3 (a) and (b) SEM images of two different regions and (c) AFM phase contrast image for the composite CE4/8 prepared from a blend electrolyte (BE38) with 4 wt \% MWCNT + 8 wt \% carbon black

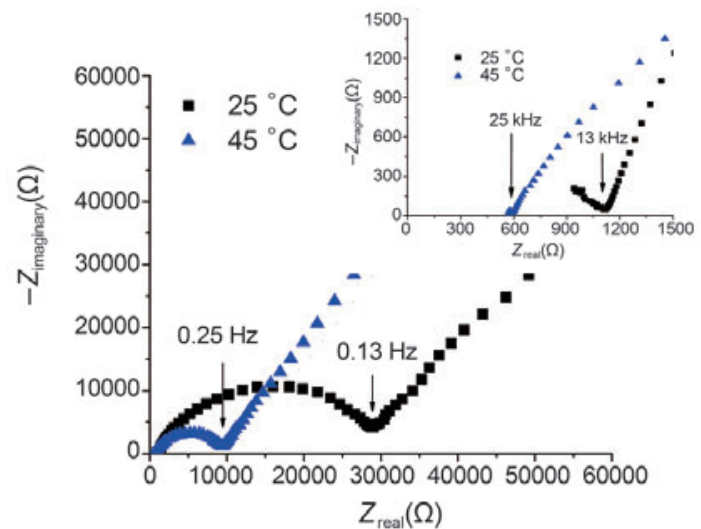

(a)

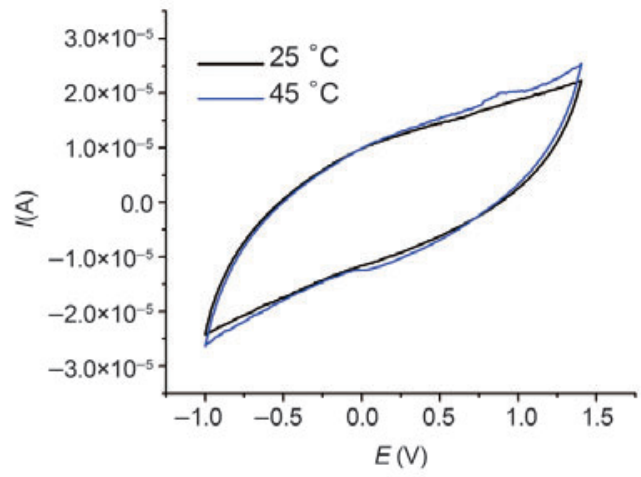

(b)

Figure 4 (a) Impedance diagrams at two magnifications and (b) cyclic voltammograms at $2 \mathrm{mV} / \mathrm{s}$ for the symmetrical polymeric multilayer capacitor prepared with BE38 electrolyte and CE4/8 electrode at two temperatures (indicated in the figure)

frequencies, and fully observed in the main Fig. 4(a), is assigned to the bulk polymer electrolyte [31]. These two components of the impedance diagram show significant changes with temperature, which indicates that the increase in ionic conduction between 25 ${ }^{\circ} \mathrm{C}$ and $45{ }^{\circ} \mathrm{C}$ also improves the overall composite conduction (ionic + electronic), probably because of the increase in the wetability of the carbonaceous materials by the polymer.

At low frequency, the straight line response is assigned to the double layer capacitance of the carbon/electrolyte interface. The capacitive behavior is clearly not the only effect observed at low frequency, because the line is not close to $90^{\circ}$. In the low-frequency range, slow ionic diffusion close to the carbonaceous material is probably changing the double layer structure. Capacitance data obtained for the device from the impedance fitting results are $1.3 \mathrm{~F} / \mathrm{g}$ at $25{ }^{\circ} \mathrm{C}$ and $2.3 \mathrm{~F} / \mathrm{g}$ at $45{ }^{\circ} \mathrm{C}$. The total mass of carbonaceous material ( $\mathrm{CB}$ and MWNT) for the two electrodes was used to calculate the specific capacitance. Although the MWNT surface area is very low, approximately $10 \%$ of the CB surface area, it can make a contribution to charge storage.

Cyclic voltammograms for the capacitor at $2 \mathrm{mV} / \mathrm{s}$ (at $25^{\circ} \mathrm{C}$ and $45{ }^{\circ} \mathrm{C}$ ) show a deviation from the boxlike shape typical of an ideal double layer capacitor [4]. Redox peaks were not clearly observed over 2.4 V. The deviation of the CV curves (Fig. 4(b)) from a box-like shape can be explained by the internal resistance and carbon porosity, which result in a current dependence of the potential [4, 7]. CV curves give the capacitances as $4 \mathrm{~F} / \mathrm{g}$ at $25^{\circ} \mathrm{C}$ and $6 \mathrm{~F} / \mathrm{g}$ at $45{ }^{\circ} \mathrm{C}$. Recent reports, based on supercapacitors with CNTs, have presented values of specific capacitances close to $20 \mathrm{~F} / \mathrm{g}$ for liquid electrolyte systems [21, 23, 32], which indicates that this "solid polymeric device" based on a nanocomposite electrode shows promising characteristics.

A specific capacitance value of $17 \mathrm{~F} / \mathrm{g}$ was 
obtained previously by our group for a similar EDLC, with different solid electrolyte and electrode materials [16]. A comparison between flexible polymeric supercapacitors, with different electrolyte and electrode materials, was presented in our previous work [16]. The results obtained in this work are similar to those discussed previously from the point of view of the relationship between specific capacitance and mass of carbonaceous material, with the difference being that all the previous work incorporated high contents of solvents or plasticizers into the polymer electrolyte. The advantage of the present design is the dimensional stability of the multilayer polymer film produced with TPU. This allows easier processing and affords a capacitor which is able to work at higher temperatures than the liquid electrolyte-based ones. Further improvements in the current collector/nanocomposite contact should be pursued. We are presently testing the addition of ionic liquids to the polymer electrolyte as a strategy to improve conductivity and capacitance [33].

\section{Conclusions}

A TPU blend and a mixture of carbon black and MWNT can be employed to prepare flexible electrolyte and electrode films. The spray coating technique permits reproducible preparation of coatings, which are easy to assemble. The low content of active material used in the electrode allows a good dispersability, and gives a nanostructured arrangement with carbon nanotubes connecting the CB aggregates. Such a supercapacitor, without leakage and flammability problems, but showing higher capacitance, should be a very important option in the spectrum of storage and conversion devices available for new technologies.

\section{Acknowledgements}

This work was supported by CT EnergiaSupercapacitores, Rede Nacional de Pesquisa em Nanotubos de Carbono - Brazil, Fapemig and CNPq. The authors are grateful to Professor Tulio Matencio and Cezar Welter (Departamento de Química/ UFMG, Brazil), for discussions and suggestions concerning the treatment of the electrochemical data, and for the images provided by the Laboratório de Nanoscopia/DF/UFMG.

\section{References}

[1] Conway, B. E. Electrochemical Supercapacitors. KluwerAcademic/Plenum: New York, 1999.

[2] Kotz, R.; Carlen, M. Principles and applications of electrochemical capacitors. Electrochim. Acta 2000, 45, 2483-2498.

[3] Pandolfo, A. G.; Hollenkamp, A. F. Carbon properties and their role in supercapacitors. J. Power Sources 2006, 157, 11-27.

[4] Frackowiak, E.; Beguin, F. Carbon materials for the electrochemical storage of energy in capacitors. Carbon 2001, 39, 937-950.

[5] Frackowiak, E.; Delpeux, S.; Jurewicz, K.; Szostak, K.; Cazorla-Amoros, D.; Beguin, F. Enhanced capacitance of carbon nanotubes through chemical activation. Chem. Phys. Lett. 2002, 361, 35-41.

[6] Emmenegger, C.; Mauron, P.; Sudan, P.; Wenger, P.; Hermann, V.; Gallay, R.; Zuettel, A. Investigation of electrochemical double-layer (ECDL) capacitors electrodes based on carbon nanotubes and activated carbon materials. J. Power Sources 2003, 124, 321-329.

[7] Niu, J. J.; Pell, W. G.; Conway B. E. Requirements for performance characterization of $C$ double-layer supercapacitors: Applications to a high specific-area C-cloth material. J. Power Sources 2006, 156, 725-740.

[8] Xu, B.; Wu, F.; Chen, R. J.; Cao, G. P.; Chen, S.; Wang, G. Q.; Yang, Y. S. Room temperature molten salt as electrolyte for carbon nanotube-based electric double layer capacitors. J. Power Sources 2006, 158, 773-778.

[9] Liu, H. T.; Zhu, G. Y. The electrochemical capacitance of nanoporous carbons in aqueous and ionic liquids. J. Power Sources 2007, 171, 1054-1061.

[10] Pushparaj, V. L.; Shaijumon, M. M.; Kumar, A.; Murugesan, S.; Ci, L.; Vajtai, R.; Linhardt, R. J.; Nalamasu, O.; Ajayan, P. M. Flexible energy storage devices based on nanocomposite paper. Proc. Natl. Acad. Sci. USA 2007, 104, 13574-13577.

[11] Staiti, P.; Minutoli, M.; Lufrano, F. All solid electric double layer capacitors based on Nafion ionomer. Electrochim. Acta 2002, 47, 2795-2800.

[12] Arico, A. S.; Bruce, P.; Scrosati, B.; Tarascon, J. M.; van 
Schalkwijk, W. Nanostructured materials for advanced energy conversion and storage devices. Nat. Mater.

2005, 4, 366-377.

[13] Pernaut, J. M.; Goulart, G. Electrochemical capacitor using polymer carbon composites. J. Power Sources 1995, 55, 93-96.

[14] Hashmi, S. A.; Latham, R. J.; Linford, R. G.; Schlindwein, W. S. Studies on all solid state electric double layer capacitors using proton and lithium ion conducting polymer electrolytes. J. Chem. Soc. Farad. Trans. 1997, 93, 4177-4182.

[15] Gu, H. B.; Kim, J. U.; Song, H. W.; Park, G. C.; Park, B. K. Electrochemical properties of carbon composite electrode with polymer electrolyte for electric doublelayer capacitor. Electrochim. Acta 2000, 45, 1533-1536.

[16] Lavall, R. L.; Borges, R. S.; Calado, H. D. R.; Welter, C.; Trigueiro, J. P. C.; Rieumont, J.; Neves, B. R. A.; Silva, G. G. Solid state double layer capacitor based on a polyether polymer electrolyte blend and nanostructured carbon black electrode composites. J. Power Sources 2008, 177, 652-659.

[17] Gauthier, M.; Belanger, A.; Bouchard, P.; Kapfer, B.; Ricard, S.; Vassort, G; Armand, A.; Sanchez, J. Y.; Krause, L. Large lithium polymer battery development-The immobile solvent concept. J. Power Sources 1995, 54, 163-169.

[18] Huang, J. C. Carbon black filled conducting polymers and polymer blends. Adv. Polym. Technol. 2002, 21, 299-313.

[19] Dresselhaus, M. S.; Avouris, P. Introduction to carbon materials research. Top. Appl. Phys. 2001, 80, 1-9.

[20] Niu, C. M.; Sichel, E. K.; Hoch, R.; Moy, D.; Tennent, H. High power electrochemical capacitors based on carbon nanotube electrodes. Appl. Phys. Lett. 1997, 70, 14801482.

[21] Du, C. S.; Yeh, J.; Pan, N. High power density supercapacitors using locally aligned carbon nanotube electrodes. Nanotechnology 2005, 16, 350-353.

[22] Futaba, D. N.; Hata, K.; Yamada, T.; Hiraoka, T.; Hayamizu, Y.; Kakudate, Y.; Tanaike, O.; Hatori, H.; Yumura, M.; lijima, S. Shape-engineerable and highly densely packed single-walled carbon nanotubes and their application as super-capacitor electrodes. Nat. Mater. 2006, 5, 987-994.

[23] Zhang, H.; Cao, G. P.; Yang, Y. S. Electrochemical properties of ultra-long, aligned, carbon nanotube array electrode in organic electrolyte. J. Power Sources 2007, $172,476-480$.
[24] dos Santos, A. S.; Leite, T. D. N.; Furtado, C. A.; Welter, C.; Pardini, L. C.; Silva G. G. Morphology, thermal expansion, and electrical conductivity of multiwalled carbon nanotube/epoxy composites. J. Appl. Polym. Sci. 2008, 108, 979-986.

[25] Trigueiro, J. P. C.; Silva, G. G.; Lavall, R. L.; Furtado, C. A.; Oliveira, S.; Ferlauto, A.S.; Lacerda, R. G.; Ladeira, L. O.; Liu, J. W.; Frost, R. L.; George, G. A. Purity evaluation of carbon nanotube materials by thermogravimetric, TEM, and SEM methods. J. Nanosci. Nanotechnol. 2007, 7, 3477-3486.

[26] Arepalli, S.; Nikolaev, P.; Gorelik, O.; Hadjiev, V. G.; Bradlev, H. A.; Holmes, W.; Files, B.; Yowell, L. Protocol for the characterization of single-wall carbon nanotube material quality. Carbon 2004, 42, 1783-1791.

[27] Bassi, M.; Tonelli, C.; di Meo, A. Glass transition behavior of a microphase segregated polyurethane based on PFPE and IPDI. A calorimetric study. Macromolecules 2003, 36, 8015-8023.

[28] Machado, J. C.; Silva, G. G.; de Oliveira, F. C.; Lavall, R. L.; Rieumont, J.; Licin, P.; Windmoller, D. Free-volume and crystallinity ion low molecular weight poly(ethylene oxide). J. Polym. Sci. B: Polym. Phys. 2007, 45, 24002409.

[29] Chatterjee, T.; Krishnamoorti, R. Dynamic consequences of the fractal network of nanotube-poly(ethylene oxide) nanocomposites. Phys. Rev. E 2007, 75, 050403.

[30] Munson-McGee, S. H. Estimation of the critical concentration in an anisotropic percolation network. Phys. Rev. B 1991, 43, 3331-3336.

[31] Furtado, C. A.; de Souza, P. P.; Silva, G. G.; Matencio, T.; Pernaut, J. M. Electrochemical behavior of polyurethane ether electrolytes/carbon black composites and application to double layer capacitor. Electrochim. Acta 2001, 46, 1629-1634.

[32] Endo, M.; Kim, Y. J.; Chino, T.; Shinya, O.; Matsuzawa, Y.; Suezaki H.; Tantrakarn, K.; Dresselhaus, M. S. Highperformance electric double-layer capacitors using massproduced multi-walled carbon nanotubes. Appl. Phys. A: Mater. Sci. Process. 2006, 82, 559-565.

[33] Costa, L. T.; Lavall, R. L.; Borges, R. S.; Rieumont, J.; Silva, G. G.; Ribeiro, M. C. Polymer electrolytes based on poly(ethylene glycol) dimethyl ether and the ionic liquid 1-butyl-3-methylimidazolium hexafluorophosphate: Preparation, physico-chemical characterization, and theoretical study. Electrochim. Acta 2007, 53, 1568-1574. 